\title{
ON A THEOREM OF COHEN AND MONTGOMERY
}

\author{
MICHEL VAN DEN BERGH ${ }^{1}$
}

\begin{abstract}
In a recent paper, Cohen and Montgomery proved a conjecture of Bergman concerning the relation between the Jacobson radical and the graded Jacobson radical of a ring graded by a finite group. In their proof they made use of the theory of Hopf algebras. In this note we give a short and elementary proof of the Bergman conjecture.
\end{abstract}

Let $R$ be a $G$-graded ring, where $G$ is a finite group. Let $J(R)$ and $J_{g}(R)$ denote the Jacobson radical and the graded Jacobson radical of $R$, respectively. In [1] Bergman conjectured that $J_{g}(R) \subset J(R)$. This conjecture is nicely complemented by the question of whether $J(R)=J_{g}(R)$ if $|G|^{-1} \in R$. Both questions are answered affirmatively by Cohen and Montgomery in [2]. In their paper they used the observation that $R$ is $G$-graded if and only if $R$ is a $k[G]^{*}$ module algebra. (In a Hopf algebra context, $k$ is always a small commutative ring such that everything that is used has a $k$-structure.) In this note we use $k[G]$ comodule algebras instead of $k[G]^{*}$ module algebras. This allows us to give a very short proof of Bergman's conjecture. We also obtain a short proof for $J(R)=J_{g}(R)$ if $|G|^{-1} \in R$. For any Hopf algebra $H$ a ring $R$ is an $H$ comodule algebra if there exists an algebra homomorphism $\rho: R \rightarrow R \otimes H$ satisfying

(1) $(\mathrm{id} \otimes \varepsilon) \circ \rho=\mathrm{id}$,

(2) $(\rho \otimes \mathrm{id}) \circ \rho=(\mathrm{id} \otimes \Delta) \circ \rho$.

Now it is elementary that $R$ is $G$-graded $\Leftrightarrow R$ is a $k[G]$-comodule algebra, where the map $\rho$ is given by

$$
\rho: R \rightarrow R \otimes k G: r \rightarrow \sum_{x \in G} r_{x} \otimes x
$$

In the rest of this note we will identify $R \otimes k G$ with $R G$, which will be considered to be graded in the usual way. We will denote the image of $R$ under $\rho$ by $S$.

The author wishes to thank Professor Passman, who gave some verv helpful remarks after reading the first version of this note.

(1) ThEOREM. $J_{g}(R) \subset J(R)$.

Proof. Let $V$ be an irreducible $R$-module. Then $W=V^{G}=\sum_{\sigma \in G} \sigma V$ is clearly a graded irreducible $R G$-module. Since $R G$ is finitely generated, as $S$-module this

Received by the editors December 2, 1983 and, in revised form, October 15, 1984.

1980 Mathematics Subject Classification. Primary 16A21, 16A26.

${ }^{1}$ The author is supported by an NFWO-grant. 
implies that $W$ is finitely generated as $S$-module. The graded version of Nakayama's lemma now implies that $J_{g}(S) W \neq W$. Set $J_{g}(R)=\sum_{\sigma \in G} I_{\sigma}$. Then $J_{g}(S)=\sum_{\sigma \in G} \sigma I_{\sigma}$. So we see that

$$
W \neq J_{g}(S) W=\left(\sum \sigma I_{\sigma}\right) W=\left(\sum I_{\sigma}\right) W=J_{g}(R) W .
$$

Since $J_{g}(R) W$ is clearly a graded submodule of $W$, then $J_{g}(R) W=0$, and hence, $J_{g}(R) V=0$. This implies that $J_{g}(R) \subset J(R)$.

Now we will prove the opposite inclusion $J(R) \subset J_{g}(R)$ if $|G|^{-1} \in R$.

(2) LEMma (Graded version of Maschke's theorem). Suppose $|G|^{-1} \in R$. If $M, N$ are two graded $R G$-modules, then there is a map $\sim \operatorname{Hom}_{S}(M, N) \rightarrow \operatorname{Hom}_{R G}(M, N)$ satisfying the following conditions:

(a) The image of a map of degree zero is a map of degree zero.

(b) If $f \in \operatorname{Hom}_{S}(M, N)$ is RG-linear, then $\tilde{f}=f$.

(c) Suppose $M^{\prime}$ and $N^{\prime}$ are two other $R G$-modules. If there is a commutative diagram of $R G$-modules

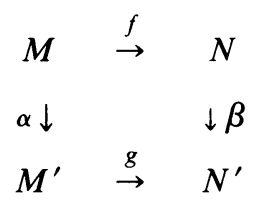

where $\alpha, \beta$ are $R G$-linear and $f, g$ are $S$-linear, then the diagram

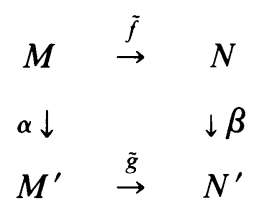

is also commutative.

Proof. Let $f \in \operatorname{Hom}_{S}(M, N), m \in M$, and define

$$
\tilde{f}(m)=\frac{1}{|G|} \sum_{\sigma \in G} \sigma f\left(\sigma^{-1} m\right) .
$$

The demonstration that $\tilde{f}$ is $R G$-linear is entirely classical. Let $\tau \in G$. Then

$$
\tilde{f}(\tau m)=\frac{1}{|G|} \sum_{\sigma \in G} \sigma f\left(\sigma^{-1} \tau m\right)=\frac{1}{|G|} \sum_{\sigma^{\prime} \in G} \tau \sigma^{\prime} f\left(\sigma^{\prime-1} m\right)=\tau \tilde{f}(m) .
$$

It is obvious that $\tilde{f}$ satisfies properties (a)-(c).

(3) COROLLARY. Let $V$ be a graded RG-module that is graded completely reducible as $S$-module. Then $V$ is graded completely reducible as $R G$-module.

Proof. Again, this is entirely classical. Let $W$ be a graded $R G$-submodule of $V$. Then the inclusion map $W \rightarrow V$ splits as a map of $S$-modules. Hence, by Lemma (2), $W \rightarrow V$ splits as a map of $R G$-modules. 
If $\tau \in G$ and $M$ is a graded $S$-module, then $M(\tau)$ is defined as the graded $S$-module obtained by putting $(M(\tau))_{\mu}=M_{\mu \tau}$. It is clear that the functor $M \rightarrow M(\tau)$ defines an autoequivalence on the category of graded $S$-modules.

(4) Lemma. Let $M$ be a graded $S$-module. Then $R G \otimes_{S} M \simeq \sum_{\tau \in G} M(\tau)$ as graded $S$-modules.

ProOF.

$$
R G \otimes_{S} M=\sum_{\tau} \sum_{\sigma} \sigma \tau^{-1} \sigma^{-1} \otimes M_{\sigma}
$$

Now it is easy to check that $\sum_{\sigma} \sigma \tau^{-1} \sigma^{-1} \otimes M_{\sigma}$ is a graded $S$-module. The map

$$
\sum_{\sigma} \sigma \tau^{-1} \sigma^{-1} \otimes M_{\sigma} \rightarrow M(\tau): \sum \sigma \tau^{-1} \sigma^{-1} \otimes m_{\sigma} \rightarrow \sum m_{\sigma}
$$

is a graded isomorphism of $S$-modules.

(5) TheOREM. If $|G|^{-1} \in R$, then $J(R) \subset J_{g}(R)$.

PROOF. The proof is an imitation of the proof of Theorem 7.1 of [3]. Let $V$ be a graded, completely reducible $S$-module. Then $R G \otimes_{S} V$ is clearly a graded, completely reducible $S$-module, and, hence, ${ }_{R G}\left(R G \otimes_{S} V\right)$ is graded, completely reducible. In particular, $J_{g}(R G)\left(R G \otimes_{S} V\right)=0$. Let $\alpha \in J_{g}(R G)$. Then $\alpha$ may be written as $\sum_{g \in G} g \cdot s^{(g)}$. Then for any $v \in V, 0=\alpha(1 \otimes v)=\sum g \otimes s^{(g)} v$. So $s^{(g)} V=0$. Since this is true for all such $V$, we deduce that $s^{(g)} \in J_{g}(S)$, and, therefore, $J_{g}(S) G \supset J_{g}(R G)$. Taking parts of degree zero yields $J(R) \subset J_{g}(R)$.

\section{REFERENCES}

1. Bergman, Groups acting on rings, group graded rings and beyond (Preprint).

2. M. Cohen and S. Montgomery, Group graded rings, smash products and group actions, Trans. Amer. Math. Soc. 282 (1984), 237-258.

3. D. S. Passman, It's essentially Mascke's theorem, Rocky Mountain J. Math. 13 (1983), 37-54.

Department of Mathematics, UNiversity of ANTWERP, UNIVERSITEITSPlein 1, B - 2610, ANTWERP, BELGIUM 